\title{
Skeletal muscle dysfunction in COPD: relevance of nutritional support and pulmonary rehabilitation
}

In this focused issue titled "Nutritional status and muscle dysfunction in chronic obstructive pulmonary disease: stable versus acute exacerbations", several review articles have been published to discuss relevant topics related to skeletal muscle dysfunction in patients with COPD. In a first review article, Drs. Gea, Sancho-Muñoz, and Chalela have comprehensively reviewed the implications and relationships between nutritional status and skeletal muscle dysfunction in patients with COPD, both in stable conditions and during acute pulmonary exacerbations. In a second review article, Drs. Laveneziana and Dubé have meticulously described the influence of aging and comorbidities in COPD-associated skeletal muscle dysfunction and to what extent those factors may be targeted therapeutically. In a third review, Drs. Simoes and Vogiatzis have reviewed a very interesting topic based on how muscle protein metabolism can be specifically targeted by exercise training in patients with COPD. Additionally, in a fourth review article, Drs. Rabinovich and Lakhdar have provided an excellent overview on how muscle protein anabolism and catabolism can be specifically targeted by nutritional support alone and in combination with exercise training in patients with COPD. In a fifth review article, Drs. Jones and Man and other colleagues have thoroughly discussed the role of pulmonary rehabilitation as a therapeutic strategy in COPD patients during acute exacerbations. In a sixth review, Dr. Gayan-Ramirez has comprehensively analyzed all currently available strategies in pulmonary rehabilitation and nutritional support in hospitalized patients with COPD. Finally, in a seventh review article, Drs. Barreiro and Jaitovich, the guest editors of this focus issue, have reviewed the etiologic factors and molecular mechanisms that have so far been demonstrated to be involved in the skeletal muscle dysfunction in COPD patients. All these factors and mechanisms were reviewed as they could be targeted therapeutically in the near future. We hope that this focused issue will serve as a practical resource to improve management of COPD patients and inspires future research in this field. We also hope that readers enjoy accessing these comprehensive reviews, which were written by leaders in each topic covered in this focused issue.

\section{Acknowledgements}

None.

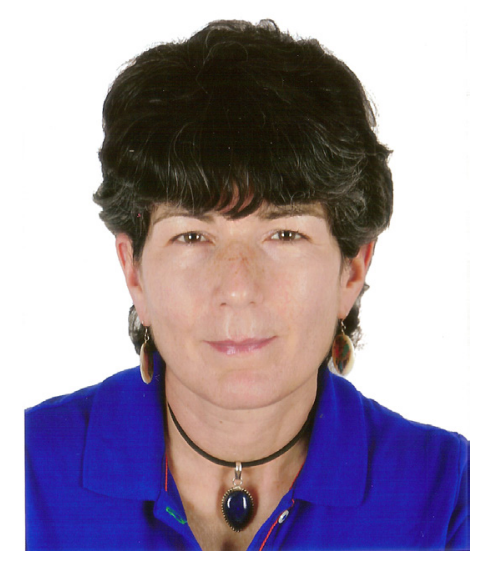

Esther Barreiro

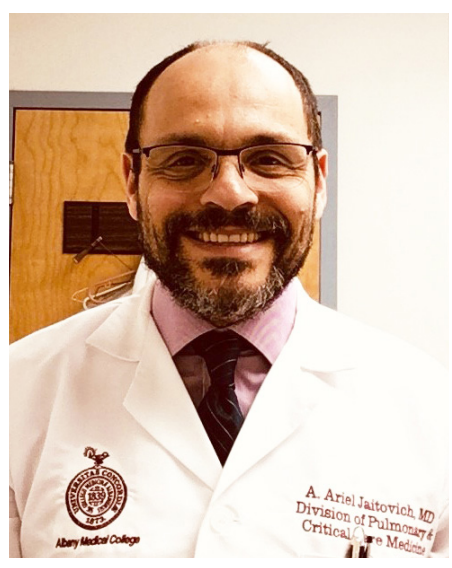

Ariel Jaitovich 


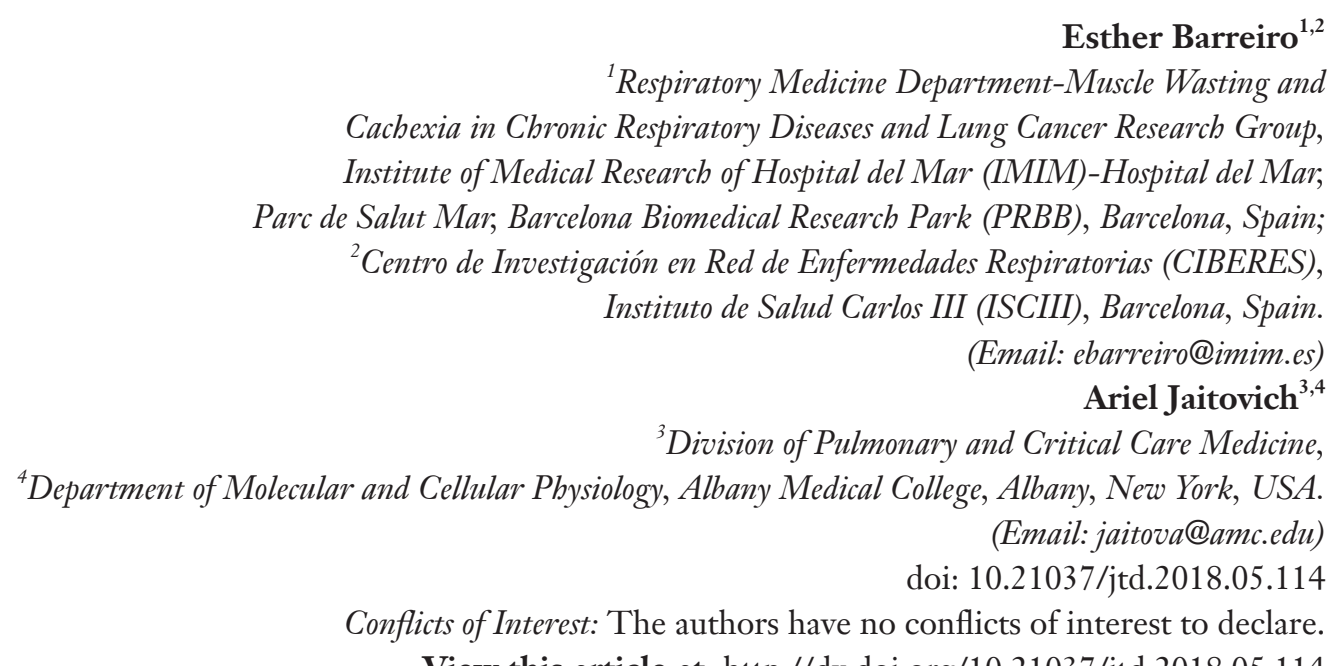

View this article at: http://dx.doi.org/10.21037/jtd.2018.05.114

Cite this article as: Barreiro E, Jaitovich A. Skeletal muscle dysfunction in COPD: relevance of nutritional support and pulmonary rehabilitation. J Thorac Dis 2018;10(Suppl 12):S1330-S1331. doi: 10.21037/jtd.2018.05.114 